\title{
Reflecting on the global burden of musculoskeletal conditions: lessons learnt from the Global Burden of Disease 2010 Study and the next steps forward
}

\author{
Damian G Hoy, ${ }^{1}$ Emma Smith, ${ }^{2,3}$ Marita Cross, ${ }^{2,3}$ Lidia Sanchez-Riera, ${ }^{4}$ \\ Fiona M Blyth, ${ }^{5}$ Rachelle Buchbinder, ${ }^{6,7}$ Anthony D Woolf, ${ }_{1}^{8}$ Tim Driscoll, ${ }^{9}$ \\ Peter Brooks, ${ }^{10}$ Lyn M March ${ }^{2,3}$
}

\begin{abstract}
Handling editor Tore K Kvien
For numbered affiliations see end of article.

\section{Correspondence to} Dr Damian G Hoy, School of Population Health, University of Queensland, Herston Rd, Herston, QLD 4006 Australia; damehoy@yahoo.com.au
\end{abstract}

Received 11 February 2014 Revised 10 May 2014

Accepted 22 May 2014 Published Online First 9 June 2014

\section{CrossMark}

\section{SLinked}

- http://dx.doi.org/10.1136/ annrheumdis-2013-204431

- http://dx.doi.org/10.1136/ annrheumdis-2013-204627

- http://dx.doi.org/10.1136/ annrheumdis-2013-204763

- http://dx.doi.org/10.1136/

annrheumdis-2013-204428

- http://dx.doi.org/10.1136/

annrheumdis-2013-204344

- http://dx.doi.org/10.1136/

annrheumdis-2013-204631

- http://dx.doi.org/10.1136/ annrheumdis-2013-204647

- http://dx.doi.org/10.1136/ annrheumdis-2013-204680

- http://dx.doi.org/10.1136/ annrheumdis-2013-204320

- http://dx.doi.org/10.1136/

annrheumdis-2014-205327

To cite: Hoy DG, Smith $\mathrm{E}$, Cross $\mathrm{M}$, et al. Ann Rheum Dis 2015;74:4-7.

\section{ABSTRACT}

The objective of this paper is to provide an overview of the strengths, limitations and lessons learned from estimating the burden from musculoskeletal (MSK) conditions in the Global Burden of Disease 2010 Study (GBD 2010 Study). It should be read in conjunction with the other GBD 2010 Study papers published in this journal. The strengths of the GBD 2010 Study include: the involvement of a MSK expert group; development of new and more valid case definitions, functional health states, and disability weights to better reflect the MSK conditions; the extensive series of systematic reviews undertaken to obtain data to derive the burden estimates; and the use of a new, more advanced version of the disease-modelling software (DisMod-MR).

Limitations include: many regions of the world did not have data; the extent of heterogeneity between included studies; and burden does not include broader aspects of life, such as participation and well-being. A number of lessons were learned. Ongoing involvement of experts is critical to ensure the success of future efforts to quantify and monitor this burden. A paradigm shift is urgently needed among global agencies in order to alleviate the rapidly increasing global burden from MSK conditions. Prevention and control of MSK disability are required, along with health system changes. Further research is needed to improve understanding of the predictors and clinical course across different settings, and the ways in which MSK conditions can be better managed and prevented.

\section{INTRODUCTION}

The Global Burden of Disease (GBD) 2010 Study is the most comprehensive effort to date for estimating the global burden of musculoskeletal (MSK) conditions. Taking almost 6 years to complete, GBD 2010 Study has estimated for the first time the burden for all MSK conditions, which include osteoarthritis (OA), rheumatoid arthritis (RA), gout, low back pain (LBP), neck pain (NP), and all other MSK disorders, captured in a group titled 'other MSK disorders'. In this article, we reflect on the strengths and limitations of GBD 2010 Study in an attempt to benefit from the lessons learned to inform future GBD studies, MSK research and interventions.

The results of GBD 2010 Study show that the prevalence and burden from MSK conditions are exceptionally high throughout the world. ${ }^{1-9}$ MSK conditions as a group cause $21.3 \%$ of the total years lived with disability (YLD) in the world, second to mental and behavioural problems (23.2\%). ${ }^{110}$ Taking into account death and disability, MSK conditions are the fourth greatest burden on the health of the world's population (third in developed countries), accounting for $6.7 \%$ of the total global disability-adjusted life years (DALY). ${ }^{1} 10$

Out of the 291 conditions studied in GBD 2010 Study, LBP ranked highest in terms of disability (YLD), and sixth in terms of overall burden (DALYs), while NP ranked fourth highest for YLD, and 21st for DALYs. OA, RA and gout were also significant contributors to the global disability burden. 'Other MSK disorders' ranked sixth highest for YLD and 23rd for DALYs.

Osteoporosis was not considered a disease in GBD 2010 Study, however, low bone mineral density (BMD) was included for the first time as one of the 67 risk factors studied. Population attributable fractions (PAF) were determined for low BMD as a risk factor for fractures, and this represented a proportion of the global burden from falls. Similarly, PAFs were determined for occupation as a risk factor for LBP, and elevated Body Mass Index as a risk factor for LBP and OA.

\section{STRENGTHS}

Previous approaches for estimating the burden of MSK conditions had four main identified limitations including: (1) estimates of incidence and duration were unreliable in some instances; (2) significant and common health states were excluded; (3) methodological heterogeneity and risk of study bias was not dealt with; and (4) there was often a paucity of suitable data. ${ }^{11} 12$ With the most recent iteration of the generic disease modelling software, DisMod-MR, and a newly established set of the disability weights (DW), GBD 2010 Study provided an opportunity to ensure that the burden of MSK conditions was quantified more accurately. As a result, the study has greatly enhanced our understanding of health burden resulting from MSK conditions.

A key strength of GBD 2010 Study was the greater involvement of content experts. This led to the formation of an international network of MSK experts who contributed to the development of new and more valid case definitions and functional health states, which better reflect the natural history of the MSK conditions. A new set of DWs was established for these health states through 
household surveys in Bangladesh, Indonesia, Peru, Tanzania, household telephone interviews in the USA, and an international web-based survey in English, Spanish and Mandarin. The surveys to establish these DWs have taken into account the importance of the geographic and socioeconomic spread of the world. The methods led to a substantially more representative and comprehensive process than in previous GBD studies, ensuring that impact on people in less developed countries is considered and included. ${ }^{11} 12$

The greatest strength of GBD 2010 Study relating to the MSK conditions was the extensive series of systematic reviews undertaken by the MSK Expert Group to obtain data to derive the burden estimates. These reviews identified a large number of population-based studies that fulfilled the set of inclusion criteria. A new risk of bias tool for prevalence studies was developed and tested. ${ }^{13}$ This tool was used to assess risk of bias of included studies. Sensitivity analyses were performed to determine the robustness of the burden estimates by excluding studies judged to be at high risk of bias.

Further significant improvement in GBD 2010 Study was the use of a new, more advanced version of the disease-modelling software (DisMod-MR). The software was able to: (1) pool heterogeneous data and adjust data for methodological differences; (2) check data on incidence, prevalence, duration, remission and mortality risk for internal consistency; (3) predict values for countries and regions with little or no data; and (4) incorporate uncertainty about the estimates in the calculations.

\section{LIMITATIONS}

Despite these strengths, there were a number of limitations that require consideration when interpreting the results of GBD 2010 Study, and planning for future GBD iterations. Many regions of the world did not have data on the prevalence of some MSK conditions. These have been identified in the disease-specific articles published in this journal. Burden estimates for these regions had to be derived through predictive modelling in DisMod-MR. As a consequence, estimates for regions with missing data are less precise and more likely to have greater uncertainty.

For some MSK conditions, such as LBP and NP, there is considerable methodological variation among population-based studies relating to the prevalence period and case definition used. Therefore, researchers are encouraged to adopt international recommendations for defining MSK conditions. ${ }^{14-18}$ This applies to MSK-specific surveys, and more importantly, to general health and disability surveys. This application will greatly enhance the validity and comparability of future burden estimates.

Another challenge was discerning the health state experience for people living with MSK conditions at the population level. Descriptions of health states were developed for GBD 2010 Study through a consensus process between the GBD MSK Expert Group and the GBD Core Team. However, while the intention was to describe the health state experience at the population level, as opposed to the clinical level, it is difficult to know with confidence how accurate these are. Further research in this area is therefore needed.

The functional domains in GBD 2010 Study refer to body functions and structures (eg, vision) as well as more complex human operations (eg, mobility). They do not refer to broader aspects of life, such as participation, well-being, carer burden and economic impact. To consider the full impact of a condition in a population, it is prudent that burden estimates are supplemented with this information.
Additionally, there was very little data available on disease severity. The data were needed to allocate the health-state DWs across each of the MSK conditions. There were sufficient disease severity data available from epidemiological studies for OA, RA and gout. However, due to a lack of data for estimating severity distribution for LBP, NP and the 'other MSK disorders', the Medical Expenditure Panel Surveys (MEP) in the USA were used. The most pertinent limitation of this is that US MEPs are very unlikely to be representative of the health-state experience for MSK conditions across the world. This is particularly the case in low-income and middle-income countries, where services for the prevention and management of MSK conditions are less extensive than in the USA, and thus, the health-state experience and distribution of severity are likely to be different from highincome countries.

Further to this, the time period over which the severity distribution was determined may be a limitation. For example, the distribution of the severity of RA was based on recent population-based data in high-income countries. As such, there is a lack of data over time, with the current figures primarily based on treated patients, including those who may be prescribed new biological treatments. The data, therefore, may underestimate the level of severity in regions that do not have access to these latest therapies, and do not allow the assessment of the severity distribution over time.

In the case of low BMD, limitations included information on osteoporotic fractures and their burden related to falls, particularly regarding long-term outcomes, not being picked up in health information systems in many regions of the world. For mortality estimates relating to low BMD, only in-hospital deaths involving hip or vertebra fracture were included. Other types of fractures were excluded because it was considered that they were less likely to lead to death. This is likely to have underestimated the burden caused by low BMD.

\section{LESSONS LEARNED AND THE NEXT STEPS FORWARD}

Throughout the process of conducting GBD 2010 Study and subsequent analysis of the findings, a number of lessons have been learned, resulting in recommendations for the way forward of future GBD studies, and MSK research and interventions.

The number of people experiencing MSK conditions in lowincome and middle-income countries is likely to increase dramatically over the coming decades. Age is one of the most common risk factors for MSK conditions. ${ }^{19}$ The ratio of older to younger people will continue to increase throughout the world ${ }^{20}$ with the greatest rate of this ratio increase taking place in less developed countries. $^{20}$ Compounding this situation is a predicted major increase in overall populations living in less developed countries. $^{21}$ By 2050, an estimated 3.5 billion people 40 years of age or older will be living in low-income and middle-income countries compared to 645 million people living in high-income countries. ${ }^{20}$ Additionally, the number of people who are obese, which is one of the major risk factors for MSK conditions, is expected to increase most dramatically in low-income and middle-income countries over the coming two decades. ${ }^{22}$

Despite the apparent prevalence differential, there are substantially more people affected by MSK conditions in low-income and middle-income countries when compared with high-income countries. Many of the risk factors associated with MSK conditions in high-income countries are also likely to become more common in low-income and middle-income countries. Work demands are extensive in subsistence communities, and studies have found activities, such as the collection of water and farming activities can increase the risk of LBP and knee pain. ${ }^{23-25}$ 
The increasing number of people affected by MSK conditions in low-income and middle-income countries is of great concern, as the impact is likely to be extreme and compound other forms of disadvantage. Health promotion and treatment services do not receive the resourcing seen in high-income countries, and health insurance and social security do not commonly exist. A large proportion of those affected are in their most productive years of life. This can have a major effect on a family's livelihood in low-income and middle-income economies, as the ability to be productive in these years is often a necessity to support younger and older family members.

The negative impact of incapacitation due to LBP and/or OA of the knee and hip is further exacerbated in rural areas where activities such as collecting water from clean water sources and farming may reduce significantly. This, in turn, may have major consequences for the rest of the family. Water-borne diseases, such as diarrhoea, and macronutritional and micronutritional deficiencies may increase as a result. Consequently, poverty may increase and further compound community health problems. Further research is needed to gain a better understanding of the 'spill-over' effects of MSK burden in these areas in order to establish comprehensive interventions to mitigate the causes.

Governments and aid programme donors have traditionally placed the bulk of their funds towards programmes addressing high-mortality communicable diseases with a more recent heightened focus on building capacity across the health system and facilitating more integrated, less verticalised systems. However, there is still very little focus and very few opportunities to focus on developing culturally appropriate programmes for the prevention and management of MSK conditions.

WHO has been measuring and monitoring the prevalence of chronic disease risk factors in developing countries through their STEPwise surveillance programme. ${ }^{26}$ Nevertheless, the focus of the programme continues to be limited to heart disease, stroke, chronic lung disease, cancer and diabetes. WHO has added optional modules for mental health, oral health, violence and injuries, but fails to recognise and include MSK conditions, despite calls to monitor this major cause of global disability. ${ }^{27}$

Many health strategies aimed at increasing physical activity to reduce the burden of the chronic diseases listed above cannot be achieved if an individual also has a mobility-limiting MSK condition. Previous calls have been made to WHO to escalate MSK conditions to a higher priority. ${ }^{27-29}$ A paradigm shift is urgently needed among global agencies, such as WHO, if we are to alleviate the rapidly increasing global burden from MSK conditions and prevent avoidable disability. Opportunities should be explored to undertake public health campaigns targeting risk factors that are shared among the chronic diseases (eg, physical inactivity and obesity), and being explicit, that they benefit MSK health as well as other non-communicable diseases (NCDs) with high mortality. This initiative as a whole should be informed through local research on the burden and perceived determinants of MSK conditions in these communities, the ways that people manage their MSK conditions, and other ways to address the overall disease burden.

In low-income and middle-income countries, it is imperative that: (1) any MSK initiative is locally owned and driven; (2) control of planning and decision making sits with the local community; (3) extensive community consultation takes place to facilitate this ownership and improve the likelihood of sustainability; and (4) the services themselves are integrated with existing policy and structures to avoid duplication and mixed public health messages, and ensure that busy staff are not diverted from their usual activities.

There is a clear need for further research to better understand the natural history of MSK conditions in low-income, middle-income and as well as high-income countries. Long-term longitudinal population-based studies that include people with MSK conditions recruited from general population, instead of specialised care centres, such as hospitals and medical centres, are needed to provide valuable information on the average duration and severity of disability from MSK conditions. For example, Public Health England is currently considering doing surveys to gather better data on morbidity of chronic/long-term conditions. Incorporating diaries to track the daily patterns of pain and consequent disability would add greater depth to this research. While they were involved in the review of the healthstate descriptions, involvement of people affected by MSK conditions in the development of the health states was limited in GBD 2010 Study, and this should be strengthened in future GBD efforts. Involving people with MSK conditions in the development of health states will strengthen the accuracy of these. Further research is also needed to better understand appropriate treatment strategies for low back and NP.

It is recommended that juvenile MSK conditions be included in future burden initiatives, as these were not captured in the other MSK category in GBD 2010 Study. Fractures due to osteoporosis were likely to be significantly underestimated due to a range of issues including: restrictions to include only hip and vertebral fractures identified from hospital admissions; poor coding due to the lack of a separate ICD code for low trauma fracture; poor recording of osteoporosis as a possible cause for the fracture; the lack of recognition that all low trauma fractures are associated with increased morbidity and mortality; and the lack of awareness that all fractures over the age of 50 years, regardless of the level of trauma, have potential association with osteoporosis and low trauma fractures. Better methods and coding are needed to adequately capture these incidents. Furthermore, the international MSK Expert Group strongly recommends that osteoporosis be considered for inclusion as a specific disease, and not as a risk factor, in future GBD studies.

OA of joints other than hips and knees should also be considered in future GBD studies, with the health-state description and disability weights related to severe OA revisited and revised. On the one hand, there was a strong push to be parsimonious with the lay descriptions of the health states to facilitate the reading of multiple health states during the household interviews. However, on the other hand, it is important to show the wide range of ways that arthritis can impact on the sufferers in terms of loss of function and limitations in physical activity. The loss of function and participation associated with severe hip and/or knee OA that would be considered for a total joint replacement, if available, was not adequately captured by the final health state, and, nor was the disability weight as high as would have been expected given the weighting of other similar conditions, such as fracture of neck of femur: short term and long term, with or without treatment.

Capturing the total burden of MSK conditions needs more valid and standardised questions in population-based health interview and examination surveys. There should be an attempt to breakdown the 'other MSK' category to provide more useful information to clinicians and policy makers. Estimates of the prevalence and burden of milder severity levels of OA, LBP, NP and other MSK conditions also need to be included and/or strengthened in future GBD studies. The entire issue of chronic MSK pain, generalised and site-specific, warrants further attention for inclusion in future burden estimates. This is because there is a growing push to include chronic pain as a disease in its own right given the enormous personal and societal impact of pain, and that MSK sites of pain are the most frequently reported chronic pain sites. 
The availability of drug treatments will affect the impact of RA, in terms of changes in disability over time and changes within different world regions. The availability of biological disease-modifying antirheumatic drugs (DMARD), most commonly in developed countries, may affect the calculated disability weights. As those receiving these treatments are likely to be considered to have 'mild' RA, subsequently, the proportions that contribute to different disability weights will vary between regions. Future research should consider assessing the impact of these treatments on the severity distribution of RA in different world regions. Likewise, assessing the impact of treatments for the other causes of MSK disorders is equally important and, therefore, also needed to be considered.

\section{CONCLUSION}

A major proportion (21\%) of global disability (YLD) is caused by MSK conditions. Ongoing involvement of experts who understand the impact of these conditions and the challenges of estimating it are critical to ensure the success of future efforts to quantify and monitor this burden. Prevention and control of MSK disability are required, along with health system changes to mitigate the growing burden of MSK conditions throughout the world. Advocacy is needed to ensure governments, donors, health service and research providers pay far greater attention to the disease burden caused by MSK conditions. ${ }^{30}$ Additionally, further research is urgently needed to improve the understanding of the predictors and clinical course of MSK across different settings, and the ways in which MSK conditions can be better managed and prevented. The lessons learned from GBD 2010 Study provide important recommendations for action.

\section{Author affiliations}

${ }^{1}$ School of Population Health, University of Queensland, Herston, Queensland, Australia

${ }^{2}$ Northern Clinical School, Institute of Bone and Joint Research, University of Sydney, St Leonards, New South Wales, Australia

${ }^{3}$ Department of Rheumatology, Royal North Shore Hospital, St Leonards, New South Wales, Australia

${ }^{4}$ Department of Rheumatology, Royal North Shore Hospital, Institute of Bone and Joint Research, University of Sydney, Australia and Institut d'Investigació Biomèdica de Bellvitge, L'Hospitalet de Llobregat, Barcelona, Spain

${ }^{5}$ School of Public Health, University of Sydney, Camperdown, New South Wales, Australia

${ }^{6}$ Department of Epidemiology and Preventive Medicine, School of Public Health and Preventive Medicine, Monash University, Victoria, Australia

${ }^{7}$ Monash Department of Clinical Epidemiology, Cabrini Hospital, Malvern, Victoria, Australia

${ }^{8}$ Department of Rheumatology, Royal Cornwall Hospital, Truro, UK

${ }^{9}$ Sydney School of Public Health, University of Sydney, Camperdown, New South Wales, Australia

${ }^{10}$ School of Population and Global Health, University of Melbourne and Northern Hospital, Parkville, Victoria, Australia

Acknowledgements We are grateful for the advice of the Chair of the GBD 2010 Study, Professor Chris Murray, and the Cluster C leader, Professor Theo Vos, at the Institute for Health Metrics and Evaluation (IHME), University of Washington, USA. We are also grateful to the many international MSK experts who willingly participated in this process.

Contributors All authors contributed to the writing and reviewing of the manuscript.

Funding Supported by the Bill and Melinda Gates Foundation (to Dr Hoy and Prof Vos), the Australian Commonwealth Department of Health and Ageing (to Dr Smith and Prof March), University of Sydney Institute of Bone and Joint Research (to Dr Smith and Dr Cross), Sociedad Española de Reumatología (to Dr Sanchez-Riera), Australian National Health and Medical Research Council (Postgraduate Scholarship 569772 to Dr Hoy and Practitioner Fellowships 334010 (2005-2009) and 606429 (2010-2014) to Prof Buchbinder, and the Ageing and Alzheimers Research Foundation (Asst Prof Blyth).

Competing interests None.
Provenance and peer review Not commissioned; externally peer reviewed.

\section{REFERENCES}

1 Murray CJ, Vos T, Lozano R, et al. Disability-adjusted life years (DALYS) for 291 diseases and injuries in 21 regions, 1990-2010: a systematic analysis for the Global Burden of Disease Study 2010. Lancet 2013;380:2197-223.

2 Cross M, Smith E, Hoy D, et al. The global burden of rheumatoid arthritis: estimates from the Global Burden of Disease 2010 study. Ann Rheum Dis 2014;73:1316-22.

3 Cross M, Smith E, Hoy D, et al. The global burden of hip and knee osteoarthritis: estimates from the Global Burden of Disease 2010 study. Ann Rheum Dis 2014;73:1323-30.

4 Hoy D, March L, Brooks P, et al. The global burden of low back pain: estimates from the Global Burden of Disease 2010 study. Ann Rheum Dis 2014;73:968-74.

5 Hoy D, March L, Woolf $A$, et al. The global burden of neck pain: estimates from the Global Burden of Disease 2010 study. Ann Rheum Dis 2014;73:1309-15.

6 Hoy D, Smith E, Cross M, et al. The global burden of musculoskeletal conditions for 2010: an overview of methods. Ann Rheum Dis 2014;73:982-9.

7 Sanchez-Riera L, Carnahan E, Vos T, et al. Global burden attributable to low bone mineral density. Ann Rheum Dis 2014;73:1635-45.

8 Smith $E$, Hoy D, Cross $M$, et al. The global burden of gout: estimates from the Global Burden of Disease 2010 study. Ann Rheum Dis 2014;73:1470-6.

9 Smith E, Hoy D, Cross M, et al. The global burden of other musculoskeleta disorders: estimates from the Global Burden of Disease 2010 study. Ann Rheum Dis 2014;73:1462-9.

10 Vos T, Flaxman AD, Naghavi M, et al. Years lived with disability (YLDs) for 1160 sequelae of 289 diseases and injuries 1990-2010: a systematic analysis for the Global Burden of Disease Study 2010. Lancet 2013;380:2163-96.

11 Murray CJ, Lopez AD, Jamison DT. The global burden of disease in 1990: summary results, sensitivity analysis and future directions. Bull World Health Organ 1994; 72:495-509.

12 Lopez AD, Mathers CD, Ezzati M, et al. Global burden of disease and risk factors. New York, NY: Oxford University Press, 2006.

13 Hoy $D$, Brooks $P$, Woolf $A$, et al. Assessing risk of bias in prevalence studies: modification of an existing tool and evidence of interrater agreement. J Clin Epidemiol 2012;65:934-9.

14 Dionne CE, Dunn KM, Croft PR, et al. A consensus approach toward the standardization of back pain definitions for use in prevalence studies. Spine 2008:33:95-103.

15 Guzman J, Hurwitz EL, Carroll LJ, et al. A new conceptual model of neck pain: linking onset, course, and care: the Bone and Joint Decade 2000-2010 Task Force on Neck Pain and Its Associated Disorders. Spine (Phila Pa 1976) 2008;33(4 Suppl):S14-23.

16 Arnett FC, Edworthy SM, Bloch DA, et al. The American Rheumatism Association 1987 revised criteria for the classification of rheumatoid arthritis. Arthritis Rheum 1988;31:315-24.

17 Kellgren JH, Lawrence JS. Radiological assessment of osteo-arthrosis. Ann Rheum Dis 1957; 16:494-502.

18 Wallace SL, Robinson H, Masi AT, et al. Preliminary criteria for the classification of the acute arthritis of primary gout. Arthritis Rheum 1977;20:895-900.

19 Hoy DG, Brooks P, Blyth F, et al. The epidemiology of low back pain. Best Pract Res Clin Rheumatol 2010;24:769-81.

20 The World Bank. Population Projections. 2011 [cited]. http://web.worldbank.org

21 United Nations Population Division. World population prospects 2010. 2011 [cited 22 September 2011]. http://esa.un.org/unpd/wpp/Excel-Data/mortality.htm

22 Kelly T, Yang W, Chen CS, et al. Global burden of obesity in 2005 and projections to 2030. Int J Obes (Lond) 2008;32:1431-7.

23 Geere JA, Hunter PR, Jagals P. Domestic water carrying and its implications for health: a review and mixed methods pilot study in Limpopo Province, South Africa. Environ Health 2010;9:52.

24 Hoy DG, Toole MJ, Morgan D, et al. Low back pain in rural Tibet. Lancet 2003;361:225-6.

25 Hoy DG, Fransen M, March L, et al. In rural Tibet, the prevalence of lower limb pain, especially knee pain, is high: an observational study. J Physiother 2010;56:49-54.

26 World Health Organisation. STEPwise approach to surveillance (STEPS). Geneva: World Health Organisation, 2013.

27 Woolf $A D$, Åkesson K. Editorial. Understanding the burden of musculoskeletal conditions. BMJ 2001;322:1079-80.

28 Woolf AD. Editorial. The Bone and Joint Decade 2000-2010. Ann Rheum Dis 2000;59:81-2.

29 Haldeman S, Kopansky-Giles D, Hurwitz EL, et al. Advancements in the management of spine disorders. Best Pract Res Clin Rheumatol. 2012;26:263-80.

30 Woolf $A D$. The Bone and Joint Decade: working together to make musculoskeleta conditions a public health priority. Arthritis Research UK Reports on the Rheumatic Diseases. 2012, Topical Reviews Series 6 no. 12. 